\title{
Anti-profibrotic effects of artesunate on bleomycin-induced pulmonary fibrosis in Sprague Dawley rats
}

\author{
CHANGMING WANG ${ }^{1}$, XIUPING XUAN $^{1}$, WENMIN YAO $^{2}$, GUOJIN HUANG $^{1,2}$ and JUNFEI JIN ${ }^{2}$ \\ ${ }^{1}$ Division of Respiratory Diseases; ${ }^{2}$ Laboratory of Hepatobiliary and Pancreatic Surgery, \\ Affiliated Hospital of Guilin Medical University, Guilin, Guangxi 541001, P.R. China
}

Received May 25, 2014; Accepted February 10, 2015

DOI: $10.3892 / \mathrm{mmr} .2015 .3500$

\begin{abstract}
The present study aimed to determine whether artesunate has beneficial effects on bleomycin-induced pulmonary fibrosis in rats and to examine the possible mechanisms underlying these effects. All experiments were performed with male Sprague Dawley rats weighing 180-250 g. Animals were randomly divided into four experimental groups that were administered either saline alone, artesunate alone, bleomycin alone or bleomycin + artesunate. Lung histopathology was investigated by hematoxylin and eosin staining and Masson staining. Lung profibrotic molecules were analyzed by reverse transcription polymerase chain reaction, immunoblotting and immunohistochemistry. In rats treated with artesunate, pulmonary fibrosis induced by bleomycin was significantly reduced. Administration of artesunate significantly improved bleomycin-induced morphological alterations. Profibrotic molecules, including transforming growth factor- $\beta 1$, Smad3, heat shock protein $47, \alpha$-smooth muscle actin and collagen type I were also reduced by artesunate. These findings suggest that artesunate improves bleomycin-induced pulmonary fibrosis pathology in rats possibly by inhibiting profibrotic molecules associated with pulmonary fibrosis.
\end{abstract}

\section{Introduction}

Pulmonary fibrosis results from different types of lung injuries and is characterized by an excessive deposition of extracellular matrix (ECM) proteins, including collagens type I-V in the alveolar wall. Pulmonary fibrosis is a potentially lethal disorder with currently no effective therapies (1). Our current

Correspondence to: Dr Changming Wang, Division of Respiratory Diseases, Affiliated Hospital of Guilin Medical University, 15 Lequn Road, Guilin, Guangxi 541001, P.R. China

E-mail:wcm@glmc.edu.cn

Dr Junfei Jin, Laboratory of Hepatobiliary and Pancreatic Surgery, Affiliated Hospital of Guilin Medical University, 15 Lequn Road, Guilin, Guangxi 541001, P.R. China

E-mail: changliangzijin@163.com

Key words: artesunate, pulmonary fibrosis, profibrotic molecules understanding of the mechanisms of pulmonary fibrosis is predominantly derived from studies of bleomycin-induced lung fibrosis in mice and rats (2-4). The underlying molecular mechanisms of lung fibrosis remain to be fully elucidated, however, certain important pathological features of pulmonary fibrosis have been identified, including increased ECM synthesis and deposition and replacement of normal functional lung tissue with an abnormal accumulation of fibroblasts and collagen (2). Certain profibrotic molecules, including transforming growth factor- $\beta 1$ (TGF- $\beta 1$ ) (3), Smad3 (4), heat shock protein 47 (HSP47) (2), $\alpha$-smooth muscle actin $(\alpha$-SMA) $(5,6)$ and collagen type I are involved in pulmonary fibrosis initiation and progression. Although significant progress has been made in understanding the molecular mechanisms of the pathogenesis of pulmonary fibrosis (7), there are no effective therapies for treating this disease and the five-year survival rate is $<50 \%$ according to clinical studies (8-10). Thus, it is necessary to identify new drugs with an improved efficacy and tolerability for pulmonary fibrosis.

Artesunate, which is recognized as being a vital cornerstone in the control of malaria (11), has been used in the treatment of severe and complicated malaria around the world $(12,13)$. Notably, a previous study demonstrated that artesunate conjugated with a single-chain variable fragment from the monoclonal antibody NP11-4 inhibited liver fibrosis induced by schistosomiasis (14). Therefore, it was hypothesized that artesunate may attenuate pulmonary fibrosis. Based on these previous findings, the present study used a rat model of pulmonary fibrosis stimulated by bleomycin instillation and treated with artesunate, to examine whether artesunate attenuates bleomycin-induced pulmonary fibrosis, and whether artesunate affects the expression of profibrotic molecules, including TGF- $\beta 1$, Smad3, HSP47, $\alpha$-SMA and collagen type I, which are important in the initiation and development of pulmonary fibrosis.

\section{Materials and methods}

Materials. Bleomycin was purchased from Zhejiang Hisun Pharmaceutical Co., Ltd. (Taizhou, China). Artesunate was purchased from Guilin Pharmaceutical Co., Ltd. (Guilin, China). The Hydroxyproline assay kit (\#A030-2) was obtained from Nanjing Jiancheng Bioengineering Institute (Nanjing, China). Primers were synthesized by Invitrogen 
Life Technologies (Shanghai, China). The cDNA synthesis kit was purchased from Takara Biotechnology Co., Ltd. (Dalian, China). The total RNA extraction kit and 2X Taq PCR Master mix were obtained from Tiangen Biotech Co., Ltd. (Beijing, China). Western blot and immunoprecipitation tissue and cell lysis solution were purchased from Beijing Biosynthesis Biotechnology Co., Ltd. (Beijing, China). The bicinchoninic acid protein assay kit was obtained from Beyotime Institute of Biotechnology (Shanghai, China). The TGF- $\beta 1$ antibody and horseradish peroxidase-conjugated goat anti-rabbit/mouse secondary antibody were purchased from Beijing Zhongshan Golden Bridge Biotechnology Co., Ltd. (Beijing, China). Smad3 antibody was purchased from Abzoom Biolabs, Inc. (Dallas, TX, USA). $\alpha$-SMA antibody and the Hsp47 antibody were purchased from Epitomics (Burlingame, CA, USA).

Animals. Male Sprague Dawley (SD) rats weighing 180-250 g (Guilin Medical University, Guilin, China) were used in all experiments. SD rats were housed in specific-pathogen-free conditions with free access to food and water.

Experimental protocols. All experimental protocols performed on all rats were approved by the Guilin Medical University Animal Experiment Ethics Committee. Animals were randomly assigned to one of the following four groups: i) Control group $(n=24)$, which received intratracheal administration of $0.9 \% \mathrm{NaCl}$ solution alone; ii) bleomycin group $(n=39)$, which received intratracheal administration of bleomycin $(5 \mathrm{mg} / \mathrm{kg})$; iii) artesunate group $(\mathrm{n}=24)$, which received daily intraperitoneal injections of artesunate $(100 \mathrm{mg} / \mathrm{kg})$ and the iv) bleomycin + artesunate group $(n=39)$, which received intratracheal administration of bleomycin $(5 \mathrm{mg} / \mathrm{kg})$ and daily intraperitoneal injection of artesunate $(100 \mathrm{mg} / \mathrm{kg})$. Bleomycin was injected into the trachea exposed through a midline anterior neck incision using a 24-gauge needle. At 7, 14, 21 or 28 days after bleomycin treatment, rats were sacrificed by decapitation under anesthesia by inhaling isofluorane $(3.5 \%$ in oxygen; sc-363629Rx; Santa Cruz Animal Health, Paso Robles, CA, USA), and lung tissue was quickly removed and processed as described below.

Histopathological analysis. Left lungs obtained from rats were instilled with $10 \%$ formalin. Tissues were embedded in paraffin and then cut into $4 \mu \mathrm{m}$ thickness sections that were used for hematoxylin and eosin (H\&E), Masson staining and immunohistochemistry analysis. The severity of interstitial fibrosis was observed and assessed by a blinded pathologist using the Ashcroft score (15). Masson staining was performed in the present study to observe collagen deposition. Right lungs obtained from rats were immediately frozen in liquid nitrogen and then stored at $-80^{\circ} \mathrm{C}$ in a freezer until use for immunoblot analysis, reverse transcription-polymerase chain reaction (RT-PCR) and hydroxyproline measurement.

Hydroxyproline measurement. The total collagen content of the right lung was determined by hydroxyproline measurement using a hydroxyproline determination kit (Nanjing Jiancheng Bioengineering Institute) according to the manufacturer's instructions. Data are expressed as milligrams of hydroxyproline per gram of protein in the lungs (mg/g).
Immunoblotting. The lung tissues were homogenized in WIP tissue and cell lysis solution with the protease inhibitor cocktail (\#04693159001; Roche Diagnostics, Basel, Switzerland) using a tissue grinder. The concentration of protein was determined using a protein assay kit (Pierce Biotechnology, Inc., Rockford, IL, USA). Approximately $30 \mu \mathrm{g}$ protein from each sample was electrophoresed in a 10 or $12 \%$ polyacrylamide gel. Following transferring proteins onto a polyvinylidene fluoride membrane (Bio-Rad Laboratories, Inc., Hercules, CA, USA), immunoblotting was performed using monoclonal rabbit anti-rat HSP47 (1:1,000 dilution; \#3198-1), TGF- $\beta 1$ (1:1,000 dilution; \#RS-0105R), $\alpha$-SMA (1:500 dilution; \#5264-1) and polyclonal rabbit anti-rat Smad3 (1:500 dilution; \#AM4061) antibodies. The proteins were visualized by incubating the membrane with chemiluminescence reagent (NEN Life Science Products, Boston, MA, USA) and exposing the membrane to X-ray films.

$R T-P C R$. RT-PCR was used in the present study to determine the mRNA expression of HSP47 and collagen type I mRNA in the lung. In brief, total RNA was isolated from the lung tissues using TRIzol reagent (Invitrogen Life Technologies, Carlsbad, CA, USA). PCR was performed using a DNA thermal cycler in a $25 \mu \mathrm{l}$ reaction volume, containing the cDNA template $(2 \mu \mathrm{l})$, forward and backward primers (1 $\mu \mathrm{l}$ each), $12.5 \mu \mathrm{l} 2 \mathrm{X}$ Master mix and $8.5 \mu 1 \mathrm{ddH}_{2} \mathrm{O}$ for 30 cycles via GeneAmp PCR system 9700 (Applied Biosystems, Foster City, CA, USA). The primer sequences were as follows: HSP47, forward 5'-AGAACCAAG GCAGACTTATCGC-3' and reverse 5'-CCGTAGATGTCC TGGTCAAAG-3'; collagen type I, forward 5'-TGCCGTGAC CTCAAGATGTG-3' and reverse 5'-CACAAGCGTGCTGTA GGTGA-3'; GAPDH, forward 5'-GTGCTGAGTATGTCG TGGAG-3' and reverse 5'-ACCAGTGGATGCAGGGAT-3'. The rat GAPDH housekeeping gene was used as an internal control.

Immunohistochemical analysis. Slides were rinsed in $1 \mathrm{X}$ phosphate-buffered saline (PBS) and then incubated in $0.3 \%$ $\mathrm{H}_{2} \mathrm{O}_{2}$ dissolved in methanol for $30 \mathrm{~min}$ in order to quench endogenous peroxidase. Following being rinsed with 1X PBS several times, slides were incubated with $3 \%$ normal serum in $1 \mathrm{X}$ PBS for $1 \mathrm{~h}$ to block nonspecific binding. Slides were then incubated with HSP47 antibody (1:400) or $\alpha$-SMA antibody (1:500) overnight at $4^{\circ} \mathrm{C}$. Following being rinsed with $1 \mathrm{X}$ PBS several times, slides were incubated with biotinylated goat anti-rabbit polyclonal secondary antibody (1:200; \#BA1000; Vector Laboratories, Burlingame, CA, USA) for $1 \mathrm{~h}$ and then avidin-biotin complex reagent (Vector Laboratories) for $30 \mathrm{~min}$. Slides were rinsed in 1X PBS and covered with diaminobenzidine peroxidase substrate solution from the Impact DAB kit (Vector Laboratories) for $2 \mathrm{~min}$ and then rinsed in water. Counterstaining was performed with hematoxylin. Slides were then dehydrated using increasing concentrations of ethanol and xylenes and mounted. Finally, images of tissue sections were captured using an Olympus BX53 digital microscope (Olympus, Tokyo, Japan).

Statistical analysis. Statistical analyses were performed with Sigmaplot software (SPSS) version 17.0 software (IBM, Armonk, NY, USA). The data are presented as the 
Table I. Artesunate reduces bleomycin-induced mortality in rats (\%).

\begin{tabular}{lcccc}
\hline Group & Day 7 & Day 14 & Day 21 & Day 28 \\
\hline Con & 0.00 & 0.00 & 0.00 & 0.00 \\
Art & 0.00 & 0.00 & 0.00 & 0.00 \\
Ble & $25.64^{\mathrm{a}}$ & 8.70 & 0.00 & 11.11 \\
Ble+Art & $20.51^{\mathrm{a}}$ & $4.00^{\mathrm{a}}$ & 0.00 & $0.00^{\mathrm{a}}$ \\
\hline
\end{tabular}

Mortality rate was the percentage of dead rats in every group at each time point. At the same time point, ${ }^{a} \mathrm{P}<0.05$ vs. the Ble group. Con, control group; Art, artesunate group; Ble, bleomycin group; Ble+Art, bleomycin+artesunate group.

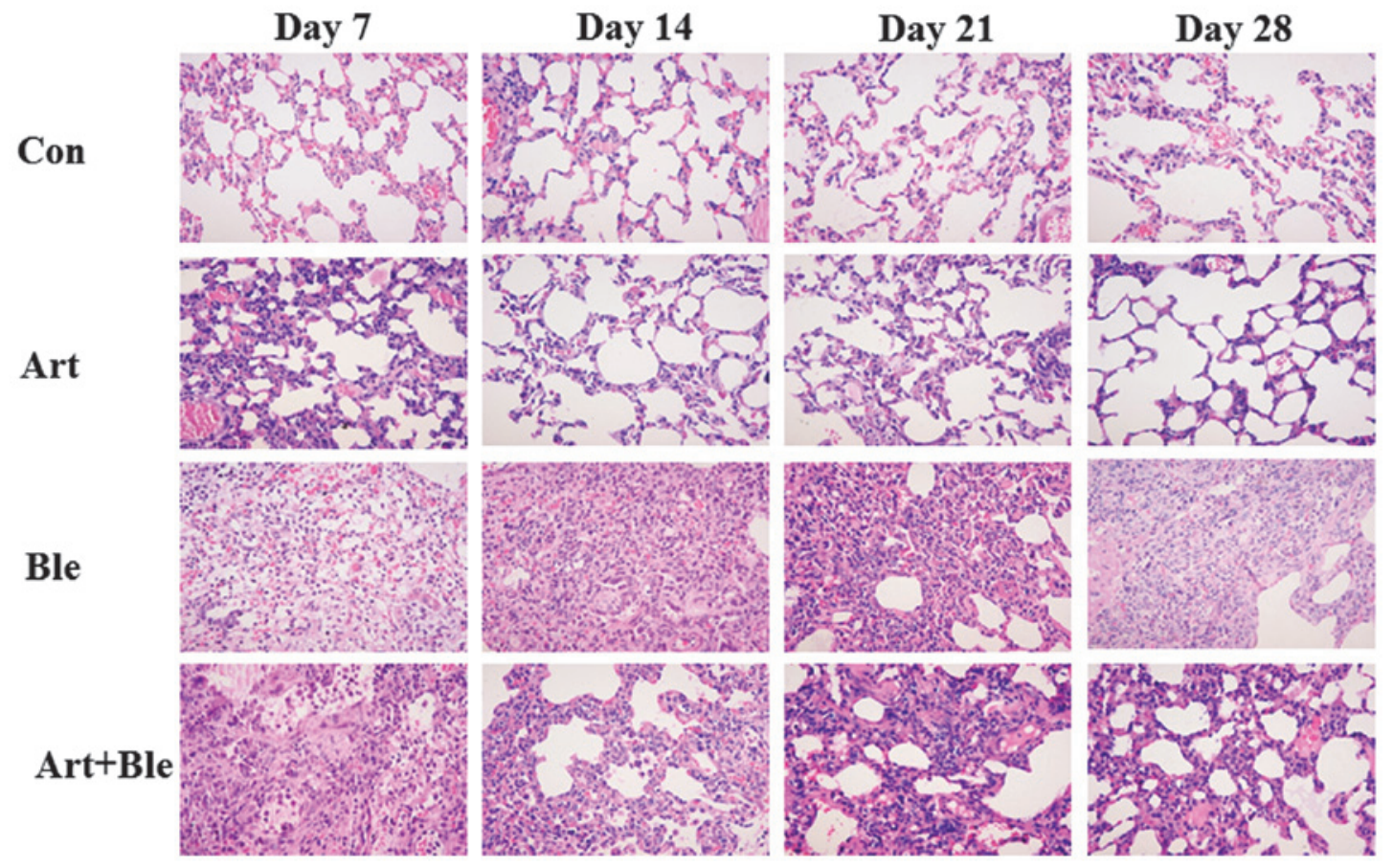

Figure 1.H\&E staining of bleomycin-treated rat lungs with and without artesunate. At 7, 14, 21 and 28 days after bleomycin injection with or without artesunate treatment, the rats were exsanguinated and their lungs were removed. Subsequently, left lower lung tissue sections were stained with H\&E. Con, control group; Ble, bleomycin group; Art, artesunate group; Ble+Art, bleomycin+artesunate group; H\&E, hematoxylin and eosin.

mean \pm standard deviation. Statistical differences were determined by two-way analysis of variance and $\mathrm{P}<0.05$ was considered to indicate a statistically significant difference.

\section{Results}

Artesunate reduces bleomycin-induced mortality in rats. As shown in Table I, $25.64,8.70$ and $11.11 \%$ of the bleomycin group rats died within 7,14 and 28 days after the administration of bleomycin, however, administration of artesunate reduced mortality to $20.51,4.00$ and $0.00 \%$ within 7,14 and 28 days, respectively. The data demonstrated that the mortality rate was highest during the first 7 days after the administration of bleomycin. Following that, the mortality rate remained at $\sim 10.00 \%$ in the bleomycin group, while the mortality rate reduced to $4.00 \%$ at 14 days. No mortality was observed between 14 and 28 days in the bleomycin+artesunate group. No mortality in the saline and artesunate group was observed during the whole experimental period. Notably, between 14 and 21 days, no mortality was found in all groups.
Artesunate attenuates bleomycin-induced pulmonary fibrosis in rats. In order to determine the pathological alterations in the lung, H\&E staining was performed (Fig. 1) and Masson staining was used to visualize the collagen fibrils (blue color) in the tissue (Fig. 2). The histological evaluation of lung sections at 7, 14, 21 and 28 days after bleomycin treatment revealed evidence of marked infiltration of inflammatory cells (particularly at day 7), excessive deposition of mature collagen in the interstitium (particularly at 14, 21 and 28 days), diffuse consolidation of parenchyma with loss of alveolar architecture and increased cell number, clear alveolar wall thickening and finally lung tissue was severely damaged. However, following artesunate treatment, the pathological alterations in the lung tissues were attenuated. The rats in the control group and artesunate alone group demonstrated no histological alterations. Similar to Masson staining, the RT-PCR results demonstrated that the mRNA expression of collagen type I (at day 28) was higher in bleomycin-treated rats compared with that in the control rats, and that the addition of artesunate then reduced this effect of bleomycin (Fig. 3). As for pulmonary 
Table II. Hydroxyproline content in the four groups at different time points $(\mu \mathrm{g} / \mathrm{g})$.

\begin{tabular}{lcccr}
\hline Group & Day 7 & Day 14 & Day 21 & Day 28 \\
\hline Con & $388.33 \pm 60.30$ & $644.00 \pm 88.60$ & $355.50 \pm 47.72$ & $435.00 \pm 65.41$ \\
Art & $424.67 \pm 37.50$ & $650.00 \pm 84.53$ & $354.67 \pm 47.58$ & $424.17 \pm 47.26$ \\
Ble & $346.80 \pm 68.10$ & $699.33 \pm 79.75$ & $513.17 \pm 72.72^{\mathrm{a}}$ & $584.50 \pm 60.85^{\mathrm{a}}$ \\
Ble+Art & $335.33 \pm 56.24$ & $719.33 \pm 108.84$ & $489.33 \pm 58.81^{\mathrm{a}}$ & $492.17 \pm 75.54^{\mathrm{b}}$ \\
\hline
\end{tabular}

At 7, 14, 21 and 28 days after bleomycin injection with or without artesunate treatment, rats were exsanguinated and their lungs were removed. Subsequently, the hydroxyproline content of the right lungs was measured using a hydroxyproline determination kit. Con, control group; Art, artesunate group; Ble, bleomycin group; Ble+Art, bleomycin+artesunate group. At the same time point, ${ }^{\mathrm{a}} \mathrm{P}<0.05$ vs. the Con and Art groups, ${ }^{\mathrm{b}} \mathrm{P}<0.05$ vs. the Ble group.

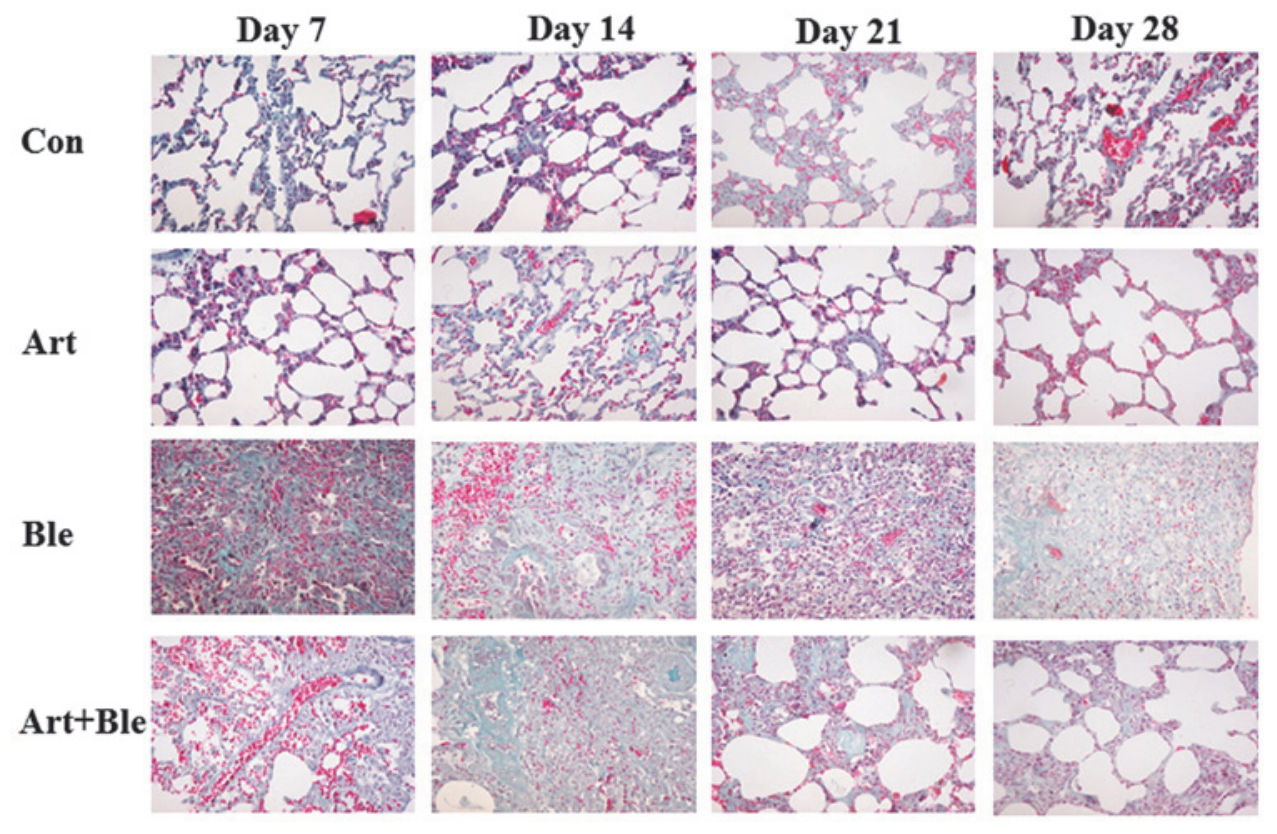

Figure 2. Masson staining of bleomycin-treated rat lungs with and without artesunate. At 7, 14, 21 and 28 days after bleomycin injection with or without artesunate treatment, the rats were exsanguinated and their lungs were removed. Subsequently, left lower lung tissue sections were stained with Masson staining. Con, control group; Art, artesunate group; Ble, bleomycin group; Ble+Art, bleomycin+artesunate group.

\section{Con Art Ble Art+Ble

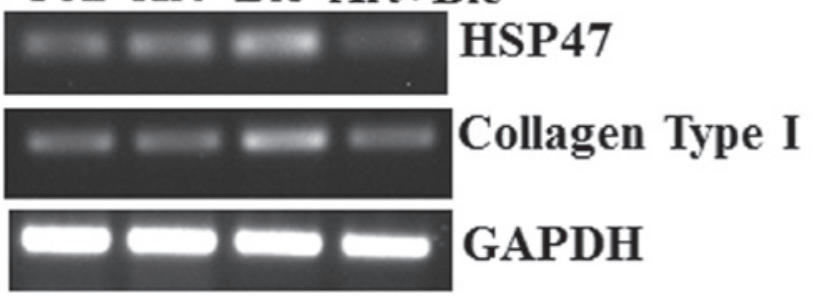

Figure 3. Lung HSP47 and collagen type I mRNA expression is reduced following artesunate treatment. At 28 days after bleomycin injection with or without artesunate treatment, the rats were exsanguinated and their lungs were removed. Reverse transcription polymerase chain reaction was performed to detect HSP47 and collagen type I mRNA expression in the lung tissues. Con, control group; Art, artesunate group; Ble, bleomycin group; Both, bleomycin+artesunate group; HSP47, heat shock protein 47.

fibrosis evaluation, the hydroxyproline content was measured. On days 7 and 14, the hydroxyproline contents were similar between the four groups. Out of all time points, the highest hydroxyproline contents were observed at day 14 in all groups. On day 21 , the rats receiving bleomycin demonstrated a higher hydroxyproline content than the control group. However, artesunate did not significantly reduce bleomycin-induced increases in hydroxyproline content on day 21 . On day 28 , the bleomycin group had a higher hydroxyproline content than the control group. However, artesunate significantly reduced increases in bleomycin-induced hydroxyproline content (Table II). Additionally, artesunate alone had no effect on hydroxyproline content. The severity of interstitial fibrosis among the four groups was compared using the Ashcroft score. As shown in Table III, on day 14, 21 and 28, the Ashcroft score was higher in the bleomycin group than that in the control group. Administration of artesunate could reduce bleomycin-induced increases in the Ashcroft score on day 21 and 28, but not on day 14 . In addition, artesunate alone had no effect on the Ashcroft score.

Artesunate inhibits HSP47 expression in bleomycin-induced pulmonary fibrosis. As stated above, bleomycin induced 
Table III. Ashcroft score in the four groups at different time points.

\begin{tabular}{lllr}
\hline Group & Day 14 & Day 21 & Day 28 \\
\hline Con & $2.17 \pm 0.42$ & $2.33 \pm 0.97$ & $2.99 \pm 0.59$ \\
Art & $2.22 \pm 0.47$ & $2.37 \pm 0.64$ & $3.06 \pm 0.59$ \\
Ble & $6.15 \pm 0.60^{\mathrm{a}}$ & $6.25 \pm 0.71^{\mathrm{a}}$ & $5.98 \pm 0.87^{\mathrm{a}}$ \\
Ble+Art & $6.29 \pm 0.36^{\mathrm{a}}$ & $3.72 \pm 0.92^{\mathrm{b}}$ & $4.01 \pm 1.25^{\mathrm{b}}$
\end{tabular}

At 14, 21 and 28 days after bleomycin injection with or without artesunate treatment, rats were exsanguinated and their lungs were removed. Following hematoxylin and eosin staining, the degree of lung fibrosis was measured using the Ashcroft score system. At the same time point, ${ }^{a} \mathrm{P}<0.05$ vs. the Con and Art groups, ${ }^{\mathrm{b}} \mathrm{P}<0.05$ vs. the Ble group. Con, control group; Art, artesunate group; Ble, bleomycin group; Ble+Art, bleomycin+artesunate group.
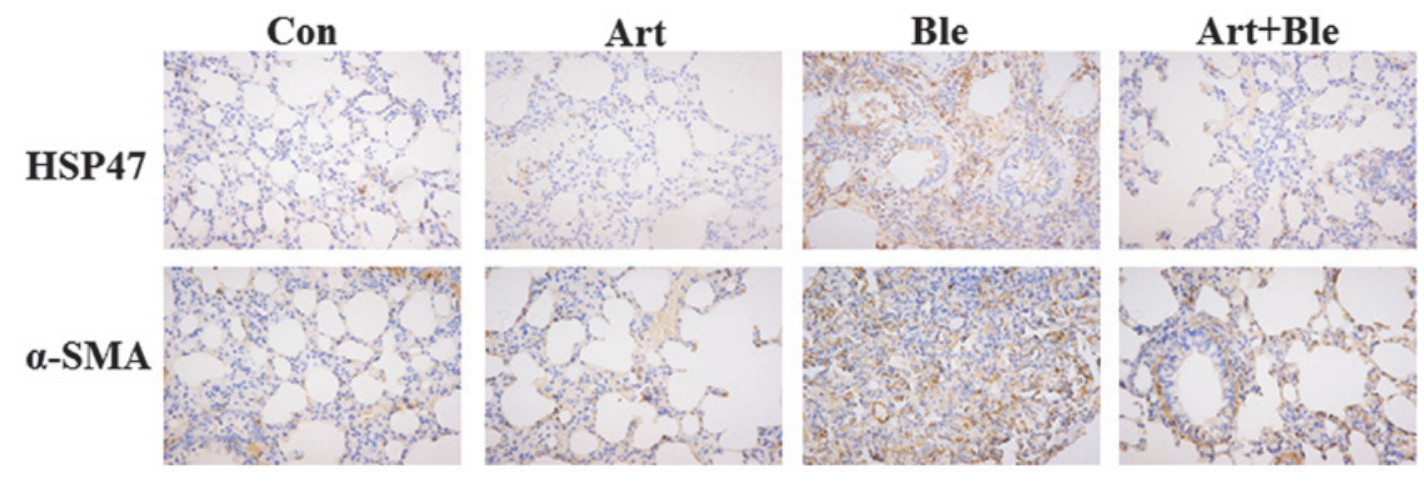

Figure 4. HSP47 and $\alpha$-SMA staining by IHC in rat lungs. At 28 days after bleomycin injection with or without artesunate treatment, rats were exsanguinated and their lungs were removed. Subsequently, left lower lung tissue sections were stained by IHC to determine HSP47 and $\alpha$-SMA expression. Con, control group; Art, artesunate group; Ble, bleomycin group; Ble+Art, bleomycin+artesunate group. HSP47, heat shock protein 47; $\alpha$-SMA, $\alpha$-smooth muscle actin; IHC, immunuhistochemistry.

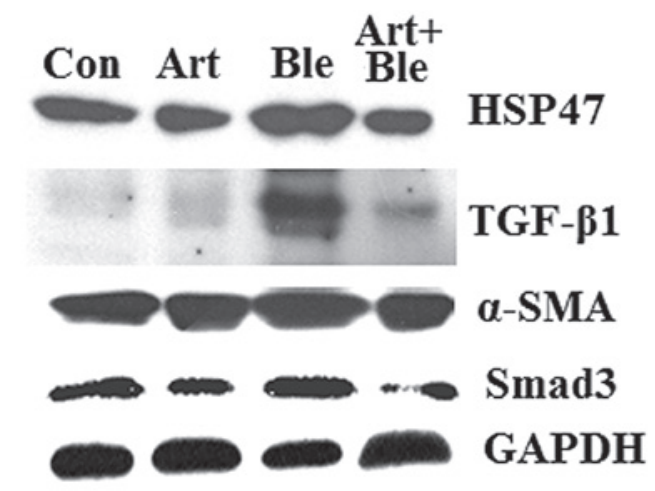

Figure 5. Western blotting to detect HSP47, TGF- $\beta 1, \alpha-$ SMA and Smad3 expression in bleomycin-treated rat lungs with and without artesunate. At 28 days after bleomycin injection with or without artesunate treatment, the rats were sacrificed and their lungs were removed. Western blotting was performed to detect HSP47, TGF- $\beta 1, \alpha-$ SMA and Smad3 protein expression in the lung tissues. Con, control group; Art, artesunate group; Ble, bleomycin group; Both, bleomycin+artesunate group; HSP47, heat shock protein 47; $\alpha$-SMA, $\alpha$-smooth muscle actin; TGF- $\beta 1$, transforming growth factor- $\beta 1$.

collagen deposition in lung tissues and artesunate inhibited this. HSP47, a collagen-binding glycoprotein, is associated with collagen accumulation and disease progression in an experimental pulmonary fibrosis model (2). Therefore, in the present study, the effect of artesunate on HSP47 was investigated. The data demonstrated that bleomycin induced HSP47 upregulation and artesunate inhibited HSP47 upregulation, which is evidenced by the results of RT-PCR (Fig. 3), immunohistochemistry (Fig. 4) and western blotting (Fig. 5).

Artesunate inhibits TGF- $\beta 1$, Smad3 and $\alpha$-SMA expression in bleomycin-induced pulmonary fibrosis in rats. TGF- $\beta 1$ and its downstream molecule $\alpha$-SMA are known to be critical for pulmonary fibrosis $(5,6)$. Western blotting confirmed enhanced protein expression of TGF- $\beta 1$ on day 28 after bleomycin injection compared with the control group (Fig. 5). In addition, Fig. 5 also shows that bleomycin-induced increases in TGF- $\beta 1$ were inhibited by artesunate. Western blotting demonstrated that bleomycin treatment led to increased Smad3 expression and artesunate administration attenuated this increase (Fig. 5). Similar to TGF- $\beta 1, \alpha-$ SMA protein was enhanced by bleomycin treatment and this enhancement was also inhibited by artesunate administration, which was confirmed by immunohistochemistry (Fig. 4) and western blotting (Fig. 5).

\section{Discussion}

As a progressive and largely untreatable group of disorders, pulmonary fibrosis is caused by exposure to radiotherapy, chemotherapeutic drugs, viral infection or other conditions. Despite numerous drugs being identified and developed to treat pulmonary fibrosis, the prognosis remains poor and new drugs with improved efficacy and tolerability are required. 
A previous study confirmed that collagen deposition must be controlled in order to reverse pulmonary fibrosis and improve the mortality rate (2). In the present study, it was found that artesunate reduced bleomycin-induced mortality in rats. Artesunate also attenuated bleomycin-induced collagen deposition, which was confirmed by H\&E staining, Masson staining, hydroxyproline content measurement, Ashcroft score evaluation and collagen type I mRNA determination by RT-PCR. Decreases in the mRNA expression of collagen type I in artesunate-treated bleomycin-induced pulmonary fibrosis rats indicated that the anti-fibrotic effect of artesunate may be mediated through direct inhibition of collagen type I expression. However, artesunate ameliorated the increases in TGF- $\beta 1$, Smad3, HSP47 and $\alpha$-SMA induced by bleomycin implying that the anti-fibrotic effect of artesunate may also be mediated by inhibition of these pro-fibrotic proteins with a resultant reduction of collagen synthesis in lung fibrosis.

TGF- $\beta 1$ is a profibrotic cytokine (3). The finding in the present study that bleomycin induced increases in TGF- $\beta 1$ protein expression, together with a previous study that TGF- $\beta 1$ expression in lung tissues was markedly elevated 21 days after bleomycin treatment (7) indicated that the upregulation of TGF- $\beta 1$ is important in the pathogenesis of bleomycin-induced pulmonary fibrosis $(16,17)$. Generally, TGF- $\beta 1$ performs its profibrotic effects by stimulation of downstream Smad protein. Smad3 was increased by bleomycin treatment in the current study. It is established that $\mathrm{Smad} 3$, a downstream protein of TGF- $\beta 1$, is necessary for TGF- $\beta 1$ signal transduction $(18,19)$. A previous study confirmed that TGF- $\beta 1 / \mathrm{Smad} 3$ affected the expression of HSP 47 in bleomycin-induced pulmonary fibrosis (4), suggesting that a TGF- $\beta 1 /$ Smad3/HSP47 signaling pathway exists affecting collagen deposition. However, a study in human lung fibroblasts revealed that TGF- $\beta 1$ induced trimer formation of heat shock factor 1 (HSF1) and then HSF1 bound to the heat shock promoter element to induce HSP47 synthesis (20), suggesting that another signaling pathway TGF- $\beta 1 /$ HSF1/HSP47 also affects collagen deposition. It is well established that HSP47 is important in the synthesis, processing and secretion of procollagen (21) and thus is important in the pathogenesis of pulmonary fibrosis. In the current study, RT-PCR, immunohistochemistry and western blotting confirmed that HSP47 expression was increased by bleomycin treatment, which is consistent with a previous study in a bleomycin model of rat pulmonary fibrosis (2). In this study, the authors found that HSP47 protein in the lung was increased, collagen accumulation and disease progression were associated with the level of HSP47 protein expression and a decrease in HSP47 expression was in line with the attenuation of fibrotic lesions and collagen expression (2). Another study revealed that the upregulated signaling pathway of TGF- $\beta 1 / \mathrm{HSP} 47 /$ collagen I may be associated with the pathogenesis of fibrosis in rats (22). $\alpha$-SMA, a downstream molecule of TGF- $\beta 1$, is known to be critical for pulmonary fibrosis $(5,6)$. HSP47 mRNA was found to be localized in $\alpha$-SMA-positive myofibroblasts in the active fibrotic areas (23), however, the precise association between HSP47 and $\alpha$-SMA remains to be elucidated.

Taken together, it was hypothesized that bleomycin affects collagen deposition through the TGF $\beta 1 \rightarrow \operatorname{Smad} 3$ (or HSF1) $\rightarrow$ HSP47 (or $\alpha$-SMA) $\rightarrow$ collagen I signaling pathway.
As confirmed in the present study, bleomycin treatment induced these increases in pro-fibrotic molecules. Notably, artesunate could inhibit bleomycin-induced pro-fibrotic molecule enhancement. Therefore, the anti-fibrotic effect of artesunate may be associated with alterations in these pro-fibrotic molecules. Thus, targeting these pro-fibrotic molecules presents a promising method for the therapy of pulmonary fibrosis. To the best of our knowledge, the present study is the first experimental study investigating the effects of artesunate in pulmonary fibrosis. The optimal effective and tolerable dosage of artesunate in pulmonary fibrosis remains to be elucidated and requires investigation in future studies. Our findings demonstrated that artesunate inhibits pulmonary fibrosis induced by bleomycin and its anti-fibrotic effects are possibly associated with the attenuation of certain pro-fibrotic proteins, including TGF- $\beta 1$, Smad3, HSP47, $\alpha$-SMA and collagen type I. However, it is noteworthy that the mechanisms by which artesunate offers protection against pulmonary fibrosis are not fully understood. Although the present study confirmed that artesunate is a promising novel drug to treat pulmonary fibrosis, clinical studies should be performed to further examine the therapeutic value of artesunate in lung fibrosis.

\section{Acknowledgements}

This study was supported by the Natural Science Foundation of Guangxi (grant no. 2011GXNSFA018220).

\section{References}

1. Gharaee-Kermani M and Phan SH: Molecular mechanisms of and possible treatment strategies for idiopathic pulmonary fibrosis. Curr Pharm Des 11: 3943-3971, 2005.

2. Hagiwara S, Iwasaka H, Matsumoto S and Noguchi T: Antisense oligonucleotide inhibition of heat shock protein (HSP) 47 improves bleomycin-induced pulmonary fibrosis in rats. Respir Res 8: 37, 2007.

3. Ohashi S, Abe H, Takahashi T, Yamamoto Y, Takeuchi M, Arai H, Nagata K, Kita T, Okamoto H, Yamamoto H and Doi T: Advanced glycation end products increase collagen-specific chaperone protein in mouse diabetic nephropathy. J Biol Chem 279: 19816-19823, 2004.

4. Chen L, Wang T, Wang X, Sun BB, Li JQ, Liu DS, Zhang SF, Liu L, Xu D, Chen YJ and Wen FQ: Blockade of advanced glycation end product formation attenuates bleomycin-induced pulmonary fibrosis in rats. Respir Res 10: 55, 2009.

5. Yang $T$, Chen $M$ and Sun T: Simvastatin attenuates TGF-betal-induced epithelial-mesenchymal transition in human alveolar epithelial cells. Cell Physiol Biochem 31: 863-874, 2013

6. Ou XM, Feng YL, Wen FQ, Huang XY, Xiao J, Wang K and Wang T: Simvastatin attenuates bleomycin-induced pulmonary fibrosis in mice. Chin Med J (Engl) 121: 1821-1829, 2008.

7. Zhu T, Zhang W, Xiao M, Chen H and Jin H: Protective role of andrographolide in bleomycin-induced pulmonary fibrosis in mice. Int J Mol Sci 14: 23581-23596, 2013.

8. Tzouvelekis A, Paspaliaris V, Koliakos G, et al: A prospective, non-randomized, no placebo-controlled, phase Ib clinical trial to study the safety of the adipose derived stromal cells-stromal vascular fraction in idiopathic pulmonary fibrosis. J Transl Med 11: 171, 2013.

9. Woodcock HV, Molyneaux PL and Maher TM: Reducing lung function decline in patients with idiopathic pulmonary fibrosis: potential of nintedanib. Drug Des Devel Ther 7: 503-510, 2013.

10. Raghu G, Collard HR, Egan JJ, et al: An official ATS/ ERS/JRS/ALAT statement: idiopathic pulmonary fibrosis: evidence-based guidelines for diagnosis and management. Am J Respir Crit Care Med 183: 788-824, 2011. 
11. Atemnkeng MA, Chimanuka B, Dejaegher B, Heyden YV and Plaizier-Vercammen J: Evaluation of Artemisia annua infusion efficacy for the treatment of malaria in Plasmodium chabaudi chabaudi infected mice. Exp Parasitol 122: 344-348, 2009.

12. Nealon C, Dzeing A, Müller-Römer U, Planche T, Sinou V, Kombila M, Kremsner PG, Parzy D and Krishna S: Intramuscular bioavailability and clinical efficacy of artesunate in gabonese children with severe malaria. Antimicrob Agents Chemother 46: 3933-3939, 2002.

13. Batty KT, Le AT, Ilett KF, Nguyen PT, Powell SM, Nguyen CH, Truong XM, Vuong VC, Huynh VT, Tran QB, Nguyen VM and Davis TM: A pharmacokinetic and pharmacodynamic study of artesunate for vivax malaria. Am J Trop Med Hyg 59: 823-827, 1998.

14. Li H, Gu C, Ren Y, et al: The efficacy of NP11-4-derived immunotoxin $\mathrm{scFv}$-artesunate in reducing hepatic fibrosis induced by Schistosoma japonicum in mice. J Biomed Res 25: 148-154, 2011

15. Ashcroft T, Simpson JM and Timbrell V: Simple method of estimating severity of pulmonary fibrosis on a numerical scale. J Clin Pathol 41: 467-470, 1988.

16. Willis BC, Liebler JM, Luby-Phelps K, Nicholson AG, Crandall ED, du Bois RM and Borok Z: Induction of epithelial-mesenchymal transition in alveolar epithelial cells by transforming growth factor-beta1: potential role in idiopathic pulmonary fibrosis. Am J Pathol 166: 1321-1332, 2005.
17. $\mathrm{Cu}$ A, Ye Q, Sarria R, Nakamura S, Guzman J and Costabel U: $\mathrm{N}$-acetylcysteine inhibits TNF-alpha, sTNFR and TGF-beta1 release by alveolar macrophages in idiopathic pulmonary fibrosis in vitro. Sarcoidosis Vasc Diffuse Lung Dis 26: 147-154, 2009.

18. Bartram $\mathrm{U}$ and Speer $\mathrm{CP}$ : The role of transforming growth factor beta in lung development and disease. Chest 125: 754-765, 2004.

19. Leask A and Abraham DJ: TGF-beta signaling and the fibrotic response. FASEB J 18: 816-827, 2004.

20. Sasaki H, Sato T, Yamauchi N, et al: Induction of heat shock protein 47 synthesis by TGF-beta and IL-1 beta via enhancement of the heat shock element binding activity of heat shock transcription factor 1. J Immunol 168: 5178-5183, 2002.

21. Koide T, Asada S and Nagata K: Substrate recognition of collagen-specific molecular chaperone HSP47. Structural requirements and binding regulation. J Biol Chem 274: 34523-34526, 1999.

22. Inoue K, Naito Y, Takagi T, et al: Daikenchuto, a Kampo medicine, regulates intestinal fibrosis associated with decreasing expression of heat shock protein 47 and collagen content in a rat colitis model. Biol Pharm Bull 34: 1659-1665, 2011.

23. Kakugawa T, Mukae H, Hishikawa Y, Ishii H, Sakamoto N, Ishimatsu Y, Fujii T, Koji T and Kohno S: Localization of HSP47 mRNA in murine bleomycin-induced pulmonary fibrosis. Virchows Arch 456: 309-315, 2010. 